\title{
RESEARCH
}

Open Access

\section{Effects of metatarsal domes on plantar pressures in older people with a history of forefoot pain}

Karl B. Landorf ${ }^{12^{*}}$ (D), Claire A. Ackland ${ }^{1}$, Daniel R. Bonanno ${ }^{1,2}$, Hylton B. Menz ${ }^{2}$ and Saeed Forghany $y^{3,4}$

\begin{abstract}
Background: Forefoot pads such as metatarsal domes are commonly used in clinical practice for the treatment of pressure-related forefoot pain, however evidence for their effects is inconsistent. This study aimed to evaluate the effects on plantar pressures of metatarsal domes in different positions relative to the metatarsal heads.

Methods: Participants in this study included 36 community-dwelling adults aged 65 or older with a history of forefoot pain. Standardised footwear was used and plantar pressures were measured using the pedar ${ }^{\circledR} \mathrm{X}$ in-shoe plantar pressure measurement system. Peak pressure, maximum force and contact area were analysed using an anatomically-based masking protocol that included three forefoot mask sub-areas (proximal to, beneath, and distal to the metatarsal heads). Data were collected for two different types of prefabricated metatarsal domes of different densities (Emsold metatarsal dome and Langer PPT metatarsal pad) in three different positions relative to the metatarsal heads. Seven conditions were tested in this study: (i) control (no pad) condition, (ii) Emsold metatarsal dome positioned $5 \mathrm{~mm}$ proximal to the metatarsal heads, (iii) Emsold metatarsal dome positioned in-line with the metatarsal heads, (iv), Emsold metatarsal dome positioned $5 \mathrm{~mm}$ distal to the metatarsal heads, (v) Langer PPT metatarsal pad positioned $5 \mathrm{~mm}$ proximal to the metatarsal heads, (vi) Langer PPT metatarsal pad positioned in-line with the metatarsal heads, and (vii) Langer PPT metatarsal pad positioned $5 \mathrm{~mm}$ distal to the metatarsal heads.
\end{abstract}

Results: When analysed with the mask that was distal to the metatarsal heads, where the plantar pressure readings were at their highest, all metatarsal dome conditions led to significant reductions in plantar pressure at the forefoot compared to the control (no pad) condition $\left(F_{3.9,} 135.6=8.125, p<0.001\right)$. The reductions in plantar pressure were in the order of 45-60 kPa. Both the Emsold metatarsal dome and the Langer PPT metatarsal pad, when positioned proximal to the metatarsal heads, managed to achieve this without adversely increasing plantar pressure proximally where the pad was positioned, however the Emsold metatarsal dome was most effective.

(Continued on next page)

\footnotetext{
* Correspondence: k.landorf@latrobe.edu.au

'Discipline of Podiatry, School of Allied Health, Human Services and Sport, La

Trobe University, Melbourne, Victoria 3086, Australia

${ }^{2}$ La Trobe Sport and Exercise Medicine Research Centre, La Trobe University, Melbourne, Victoria 3086, Australia

Full list of author information is available at the end of the article
}

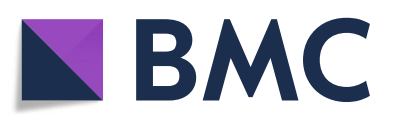

(- The Author(s). 2020 Open Access This article is licensed under a Creative Commons Attribution 4.0 International License, which permits use, sharing, adaptation, distribution and reproduction in any medium or format, as long as you give appropriate credit to the original author(s) and the source, provide a link to the Creative Commons licence, and indicate if changes were made. The images or other third party material in this article are included in the article's Creative Commons licence, unless indicated otherwise in a credit line to the material. If material is not included in the article's Creative Commons licence and your intended use is not permitted by statutory regulation or exceeds the permitted use, you will need to obtain permission directly from the copyright holder. To view a copy of this licence, visit http://creativecommons.org/licenses/by/4.0/. The Creative Commons Public Domain Dedication waiver (http://creativecommons.org/publicdomain/zero/1.0/) applies to the data made available in this article, unless otherwise stated in a credit line to the data. 
(Continued from previous page)

Conclusions: Metatarsal domes reduce plantar pressure in the forefoot in older people with a history of forefoot pain. All metatarsal dome conditions significantly reduced peak pressure in the forefoot, however metatarsal domes that were positioned $5 \mathrm{~mm}$ proximal to the metatarsal heads provided the best balance of reducing plantar pressure distal to the metatarsal heads, where the pressure is at its greatest, but not adversely increasing plantar pressure proximally, where the bulk of the pad is positioned. In this proximal position, the Emsold metatarsal dome was more effective than the Langer PPT metatarsal pad and we cautiously recommend this forefoot pad for alleviating forefoot pressure in older people with forefoot pain.

Keywords: Aged, Pain, Forefoot, human, Orthoses, Orthotic devices, Biomechanics, Kinetics, Plantar pressure

\section{Introduction}

Foot pain is a common complaint in older people - it is estimated that between 20 and $29 \%$ of older people have foot pain [1-3]. Further, foot pain in older people can affect mobility, gait and balance $[4,5]$. One region of the foot that is commonly affected by pain is the forefoot [6], which is defined by pain in the region of the metatarsals heads $[7,8]$. In older people, forefoot pain is the most common type of foot pain, accounting for approximately $37 \%$ of all foot pain [6].

Forefoot pain in older people is associated with many causes, including high plantar pressures under the forefoot $[4,9]$. Therefore, redistribution of high forefoot plantar pressures using forefoot pads may reduce forefoot pain [10]. There are many different types of forefoot pads, although a commonly used type is the metatarsal dome, a teardrop shaped pad that is usually positioned just proximal to the middle metatarsal heads [11]; although, the exact position of metatarsal domes has not been clearly defined and evaluated.

We previously tested the plantar pressure redistribution properties of forefoot pads, including a metatarsal dome that was tested in two positions $(10 \mathrm{~mm}$ proximal and $5 \mathrm{~mm}$ distal to the metatarsal heads) [12]. We found metatarsal domes in both positions reduced forefoot peak pressure $(9 \%$ reduction with the proximal dome and $17 \%$ reduction with the distal dome), however we could not accurately determine where in the forefoot peak pressure was reduced, nor could we adequately explain how the metatarsal domes achieved their effect.

Currently, there is limited data on the effect of metatarsal domes on forefoot plantar pressures. Further, the optimal position of a metatarsal dome for redistributing plantar pressure is currently unknown [13-15]. The aim of this study was to measure the effect of different metatarsal domes in different positions on plantar pressures in older people with a history of forefoot pain.

\section{Methods}

\section{Ethics approval}

Ethics approval was obtained from the La Trobe University Faculty Human Ethics Committee - application FHEC12/
207. All participants signed written informed consent prior to recruitment into the study.

\section{Participants}

The participants were 36 community-dwelling older adults from Melbourne, Australia.

\section{Inclusion criteria}

Participants were eligible if they:

(i) were aged 65 years or older;

(ii) were community-dwelling;

(iii) had forefoot pain or a previous history of forefoot pain;

(iv) were able to walk household distances $(10 \mathrm{~m})$ without the use of a walking aid;

(v) were cognitively aware, so they could understand the requirements of the project;

(vi) were able to speak basic English, so they could provide informed consent prior to participation, follow instructions during the project, and to answer research questions accurately.

\section{Exclusion criteria}

Participants were excluded from the study if they:

(i) had any self-reported condition that may have affected lower limb sensation or muscle strength such as a stroke, polio, diabetic peripheral neuropathy;

(ii) had any lower limb surgery in the previous 3 months;

(iii) had any lower limb amputations that may affect lower limb function.

\section{Recruitment}

All participants were recruited from a study population involved in a previous clinical trial $[16,17]$. Participants in this study were only recruited after they had completed all requirements in the clinical trial.

\section{Sample size determination}

The sample size was determined prior to conducting the study using an appropriate formula [18]. A sample size 
of 36 provides an $80 \%$ probability of detecting a clinically meaningful difference between interventions of $60 \mathrm{kPa}$ in peak plantar pressure. The standard deviation used to determine this sample size was taken from a similar study that measured plantar pressures in older people [19] and was set at $90 \mathrm{kPa}$. The alpha level was set at 0.05 .

\section{Setting}

The study was performed in a research room in the Health Sciences Clinic at La Trobe University in Melbourne, Australia.

\section{Interventions}

Two different brands of prefabricated metatarsal domes (i.e. pads) were used: (i) Emsold metatarsal dome (Emsold-Gesellschaft Gert Helmers GmbH \& Co. KG, Rastede, Germany), and (ii) Langer PPT metatarsal pad (Langer Biomechanics, Ronkonkoma, New York, USA). Each dome differs in its hardness; the Emsold metatarsal dome has an average Shore A hardness of 11 durometer, while the Langer PPT metatarsal pad is harder, with an average Shore A hardness of 20 durometer. Both metatarsal domes are $6 \mathrm{~mm}$ at their highest point and are teardrop shaped. The domes were dispensed in two different sizes depending on foot size. The Emsold metatarsal dome was supplied in sizes 3 and size 5, which correspond to the Langer PPT metatarsal pad sizes small and medium, respectively. The metatarsal domes were supplied free of charge by Briggate Medical Company (Braeside, Victoria, Australia).

The metatarsal domes were adhered using doublesided adhesive tape to a cardboard template (similar to an insole) sized to fit into the shoe - this prevented the pad from moving during testing. The template was positioned between the plantar surface of the foot and the bottom of the inside of the shoe. All participants were tested in a control shoe (see protocol below), so the only difference between conditions was the type of metatarsal dome (with the control condition having no dome). Each dome was tested in three positions relative to the metatarsal heads: a proximal position, an in-line position, and a distal position (Fig. 1).

Therefore, there was one control condition and six different metatarsal dome conditions assessed (i.e. seven conditions in total):

(i) shoe with cardboard template only (no pad control);

(ii) Emsold metatarsal dome positioned $5 \mathrm{~mm}$ proximal to the metatarsal heads;

(iii) Emsold metatarsal dome positioned in-line with the metatarsal heads;

(iv) Emsold metatarsal dome positioned $5 \mathrm{~mm}$ distal to the metatarsal heads;

(v) Langer PPT metatarsal pad positioned $5 \mathrm{~mm}$ proximal to the metatarsal heads;

(vi) Langer PPT metatarsal pad positioned in-line with the metatarsal heads;

(vii)Langer PPT metatarsal pad positioned $5 \mathrm{~mm}$ distal to the metatarsal heads.

The borders of the metatarsal dome conditions in relation to the anatomical landmarks of the foot are shown below in Table 1.

\section{Randomisation}

To minimise ordering effects associated with the administration of the conditions, the order of testing for each
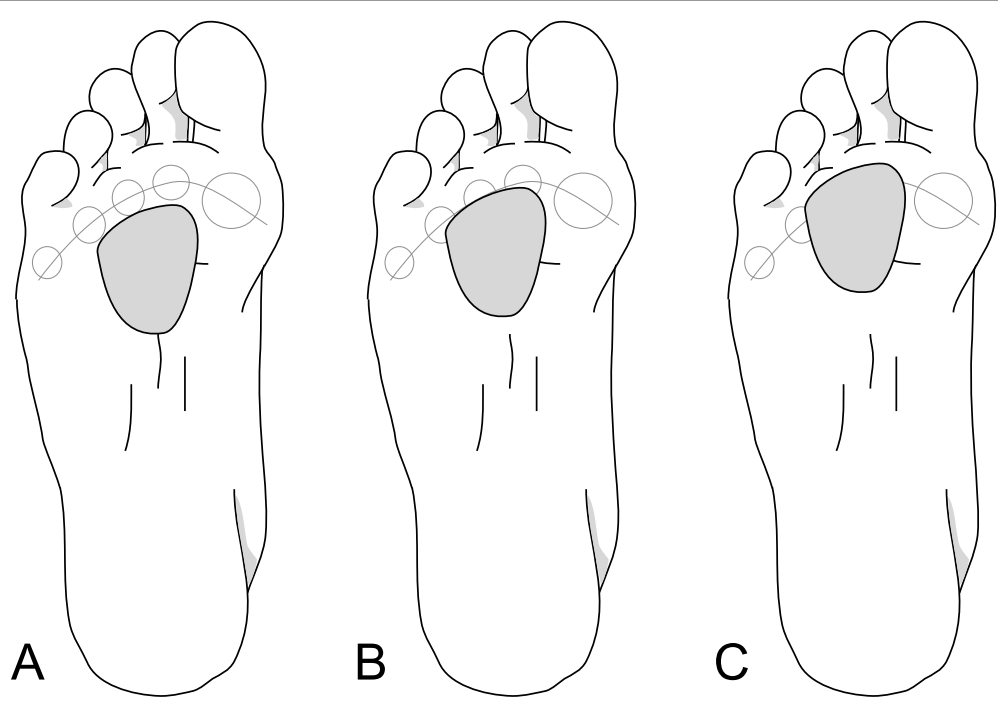

Fig. 1 The three positions that the metatarsal domes were evaluated in $(\mathbf{a}=$ proximal, $\mathbf{b}=$ in-line, $\mathbf{c}=$ distal $)$ 
Table 1 Positioning and approximate borders of the metatarsal domes

\begin{tabular}{lllll}
\hline Position tested* & Proximal border & Distal border & Medial border & Lateral border \\
\hline $\mathbf{5} \mathbf{~ m m}$ proximal to the & $\begin{array}{l}15 \mathrm{~mm} \text { distal to the styloid } \\
\text { metatarsal heads }\end{array}$ & $\begin{array}{l}5 \mathrm{~mm} \text { proximal to } \\
\text { the metatarsal heads }\end{array}$ & $\begin{array}{l}\text { Medial margin of } \\
\text { the 2nd metatarsal }\end{array}$ & $\begin{array}{l}\text { Lateral margin of } \\
\text { the 4th metatarsal }\end{array}$ \\
$\begin{array}{l}\text { In-line with the metatarsal } \\
\text { heads }\end{array}$ & $\begin{array}{l}20 \mathrm{~mm} \text { distal to the styloid } \\
\text { process of the } 5 \text { th metatarsal }\end{array}$ & $\begin{array}{l}\text { In-line with the } \\
\text { metatarsal heads }\end{array}$ & $\begin{array}{l}\text { Medial margin of } \\
\text { the 2nd metatarsal }\end{array}$ & Lateral margin of \\
the 4th metatarsal \\
$\mathbf{5} \mathbf{~ m m}$ distal to the & $25 \mathrm{~mm}$ distal to the styloid & $5 \mathrm{~mm}$ distal to the & Medial margin of & Lateral margin of \\
metatarsal heads & process of the 5 th metatarsal & metatarsal heads & the 2nd metatarsal & the 4th metatarsal
\end{tabular}

*Notes: (i) both types of metatarsal domes (Emsold and Langer) were tested in all three positions, and (ii) for full details of how the metatarsal heads were located and marked on the template see the 'Protocol' sub-section in the Methods

of the domes was randomised according to a random computer-generated sequence using Microsoft Excel ${ }^{\odot}$ 2007 (Microsoft, Redmond, WA, USA).

\section{Blinding}

The participants were blinded to the types of interventions used. They were issued a standard set of instructions informing them that they were being tested with different forefoot pads but the designs and materials used were not revealed to them. Assessor blinding was not carried out due to the difficulty in concealing the interventions. However, because the outcome measures were objective plantar pressure measurements, the lack of assessor blinding was not considered a potential source of bias.

\section{Equipment}

Plantar pressures beneath the foot were measured using the pedar $-\mathrm{X}$ in-shoe plantar pressure system (Novel GmbH, Munich, Germany). The pedar ${ }^{\circledR} \mathrm{X}$ comprises of 99 capacitive sensors arranged in a grid and embedded within a thin flexible insole. The pedar $-\mathrm{X}$ insoles were calibrated using the trublu ${ }^{\odot}$ calibration device as per the manufacturer's guidelines (Novel GmbH, Munich, Germany) prior to data collection. The sampling frequency of the system was $50 \mathrm{~Hz}$. The pedar $-\mathrm{X}$ is widely used in foot plantar pressure research [19-24] and has been demonstrated to be a valid and reliable in-shoe pressure measurement system [25-28]. It has high testretest reliability with coefficients of repeatability for metatarsal head measurements of between 1.2 to $7.7 \%$ [27], and coefficients of variation for metatarsal head measurements of between 3.4 and $24.1 \%$ [28]. This equipment has also previously been used in similar projects with older people $[12,19,29]$.

\section{Protocol}

Participants were required to attend one test session that was of approximately $75 \mathrm{~min}$ duration. Screening for inclusion into the study was initially conducted by phone when the appointment was made for the test session. Eligibility was based on self-reporting by the participant of conditions from the inclusion and exclusion criteria.
In the case of walking household distances, participants were asked this question over the phone and this was confirmed at their test session. If they were unable to walk from the waiting room to the research room where testing was conducted (a distance of $10 \mathrm{~m}$ ) without a walking aid, they were excluded.

After confirmation that the participant had read and understood the participant information statement, all participants signed the informed consent form prior to commencing the data collection session. Once informed consent was obtained, standard demographic and participant information were collected on a data collection form. Following this, participants' shoe size was determined and they were fitted with a pair of standardised, extra-depth shoes (Gadean Footwear, O'Connor, WA, Australia). The standardised shoe was used during all testing to control for influences of footwear on plantar pressures. The shoe had a sole hardness of Shore A 40 durometer, with a heel height of $27 \mathrm{~mm}$ and a forefoot height of $13 \mathrm{~mm}$.

Cardboard insoles that were matched to the size of each participant's shoes were then inserted into the shoe. The metatarsal heads and styloid process were palpated and marked with an ink pen. Next, the participant was asked to don the shoes, with the cardboard template inside. Following this, they were asked to stand up, allowing the ink to be transferred from the foot to the cardboard insole. This marked the position of each metatarsal head and the styloid process on the insole. The participant was then asked to take the shoes off so the cardboard template could be removed, thus allowing the outline of the metatarsal parabola to be marked on the insole. The three positions of the metatarsal pads (proximal, in-line, and distal) were measured on the template based on the metatarsal parabola (Table 1). The cardboard template (with or without a metatarsal dome) was then inserted back into the shoe.

Participants then had the pedar ${ }^{\circ} \mathrm{X}$ equipment attached to them and connected ready for use. The appropriately sized pedar $-\mathrm{X}$ insole was inserted into the shoe on top of the cardboard template, and the participant put the shoes on again, so the pedar $-\mathrm{X}$ insole was positioned between the participant's foot and the cardboard 
template. Participants were given sufficient time to acclimatise and be comfortable walking in the standardised shoes and with the pedar $-\mathrm{X}$ equipment in place. Next, the participant was instructed to walk at their normal comfortable speed while being timed. To minimise the confounding effect of different walking speeds on the pressure data, a trial was repeated if the walking speed for each condition differed by more than $5 \%$ of the original walking speed. Four walking trials along an $8 \mathrm{~m}$ walkway were recorded for each test condition, with the middle four steps for each trial included in the analysis (to exclude acceleration and deceleration steps). The 16 steps ( 4 trials $\times 4$ steps) were subsequently averaged for each test condition.

\section{Outcome measures}

The primary outcome measure was peak pressure under the forefoot. The secondary outcome measures were maximum force at the time of peak pressure and contact area at the time of peak pressure under the forefoot. To cross-check walking speed, total contact time for each intervention was also measured.

\section{Data analysis}

The plantar pressure data were entered into the pedar ${ }^{\circ}$ analysis program and a new, previously published anatomically-based masking protocol was used where there are three mask sub-areas of the forefoot according to their position relative to the metatarsal heads [30]. The three sub-areas were: (i) proximal to the metatarsal heads, (ii) beneath the metatarsal heads, and (iii) distal to the metatarsal heads (Fig. 2). This protocol has previously been published and was found to have excellent intra- and inter-rater reliability [30]. The reason for utilising this protocol was to enhance our analysis by being able to more precisely determine where force and contact area were being altered to achieve the plantar pressure reductions observed with the metatarsal domes. The same masking protocol was applied for each participant and for each trial to ensure consistency.

Data were analysed using IBM Statistical Package for the Social Sciences (SPSS) Version 25.0 (IBM Corporation, Armonk, NY). All data were explored for normality prior to inferential analysis. A one-way repeated-measures analysis of variance (ANOVA) with Bonferroni-adjusted posthoc tests were used to compare means between each of the conditions. Differences between conditions were considered significant if $p<0.05$. Where the data violated the assumption of sphericity in ANOVA (if $p<0.05$ for Mauchley's Test of Sphericity), the Greenhouse-Geisser correction was used to obtain the degrees of freedom and $p$-values for the $F$-statistic. proximal

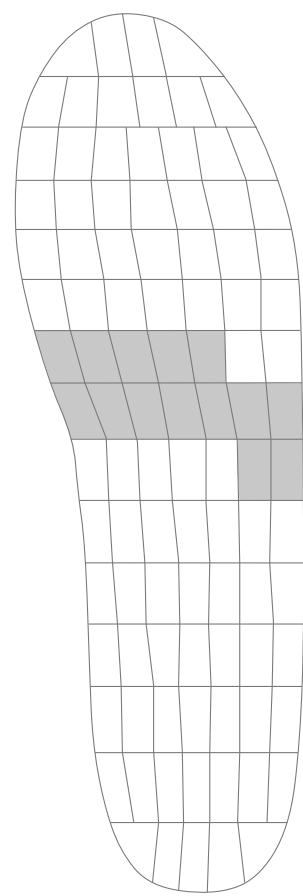

beneath

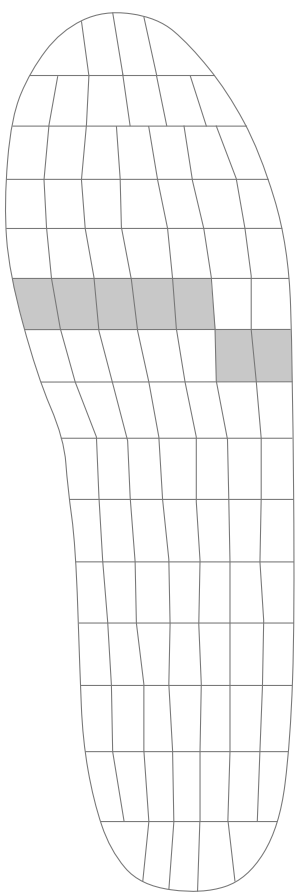

distal

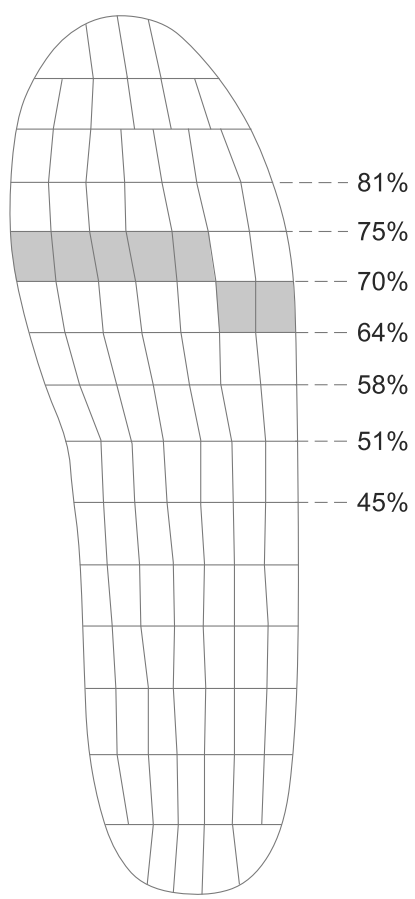

Fig. 2 Anatomically-based masking protocol used in the study (from Forghany et al. [30]) 


\section{Results}

\section{Participants}

Of the 36 participants recruited into the study, 31 were female $(86 \%)$ and 5 were male (14\%). The mean (SD) age was 75.5 (5.5) years, with a range of 65.1-88.5. Participant characteristics are shown in Table 2.

\section{Walking speed}

Because changes in walking speed can affect plantar pressures, we initially assessed whether walking speed changed between the experimental conditions by analysing for differences in contact time prior to statistical analysis. There was no significant difference for contact time between the 7 conditions $\left(\mathrm{F}_{4.5}, 157.4=1.892, p=\right.$ 0.106) indicating that the participants walked at a consistent speed for all conditions (Table 3). Therefore, any plantar pressure differences that were found can be directly attributed to the test condition, not due to changes in contact time.

\section{Peak pressure}

When analysed with the mask that was proximal to the metatarsal heads, where the bulk of the metatarsal dome was positioned, there was a significant effect for peak pressure at the forefoot between the 7 conditions $\left(\mathrm{F}_{2.1,73.5}=\right.$ $3.140, p=0.047)$. While none of the metatarsal dome conditions significantly altered peak pressure when compared to the control (no pad) condition (Table 4 and Fig. 3), there were several significant differences between the metatarsal dome conditions (Additional file 1 presents pairwise comparisons) dependent on the position of the metatarsal dome (proximal, in-line or distal). Generally, the more proximal a metatarsal dome was positioned, the lower the plantar pressure was. The proximally positioned Emsold metatarsal dome provided the lowest plantar pressure compared to the in-line and distally positioned metatarsal domes (both Emsold and Langer). However, there was no significant difference in peak pressure between the proximally positioned Emsold metatarsal dome and the proximally positioned Langer PPT metatarsal pad.

When analysed with the mask that was beneath the metatarsal heads, there was a significant effect for peak pressure at the forefoot between the 7 conditions $\left(\mathrm{F}_{2.0}\right.$, $70.4=6.713, p=0.002$ ). While none of the metatarsal

Table 2 Participant characteristics $(N=36)$

\begin{tabular}{ll}
\hline Characteristic & $\begin{array}{l}\text { Number (\%), unless } \\
\text { otherwise stated }\end{array}$ \\
\hline Age in years - mean (SD), range & $75.5(5.5), 65.1-88.5$ \\
Sex - females & $31(86 \%)$ \\
Height - mean (SD) & $1.62(0.09)$ \\
Body weight - mean (SD) & $74.3(13.4)$ \\
BMl in $\mathrm{kg} / \mathrm{m}^{2}$ - mean (SD) & $28.4(4.1)$ \\
\hline
\end{tabular}

Table 3 Contact time $(N=36)$

\begin{tabular}{lll}
\hline Condition & Mean (ms) & SD (ms) \\
\hline Control (no pad) & 702.9 & 89.4 \\
Emsold metatarsal dome proximal & 700.7 & 90.6 \\
Emsold metatarsal dome in-line & 696.0 & 82.1 \\
Emsold metatarsal dome distal & 706.5 & 92.5 \\
Langer PPT metatarsal pad proximal & 696.0 & 84.3 \\
Langer PPT metatarsal pad in-line & 701.1 & 82.6 \\
Langer PPT metatarsal pad distal & 700.8 & 91.1 \\
\hline
\end{tabular}

dome conditions significantly altered plantar pressure when compared to the control condition (Table 4 and Fig. 3), there were two significant differences between the metatarsal dome conditions (Additional file 1 presents pairwise comparisons). The in-line positioned Emsold metatarsal dome reduced plantar pressure more than the distally positioned Emsold metatarsal dome and the distally positioned Langer PPT metatarsal pad.

When analysed with the mask that was distal the metatarsal heads, where the highest plantar pressures were recorded, there was a significant effect for peak pressure at the forefoot between the 7 conditions $\left(\mathrm{F}_{3.9}\right.$, $135.6=8.125, p<0.001)$. All of the metatarsal dome conditions significantly reduced pressure when compared to the control condition (Table 4 and Fig. 3), but there were no significant differences between any of the metatarsal dome conditions (Additional file 1 presents pairwise comparisons). This reduction in plantar pressure was in the order of $45-60 \mathrm{kPa}$.

Summarising the peak pressure findings, the metatarsal domes led to significant reductions in plantar pressure at the forefoot. This reduction in plantar pressure was observed most in the mask that was distal to the metatarsal heads, where the plantar pressure readings were at their highest. For some of the metatarsal dome conditions this did not occur at the expense of increasing plantar pressure in the mask that was proximal to the metatarsal heads, where the bulk of the metatarsal dome was positioned; that is, peak plantar pressure was not simply moved from one area to another. Overall, there were no significant differences between the two metatarsal domes (i.e. Emsold metatarsal dome and Langer PPT metatarsal pad) in their ability to reduce plantar pressure when both were positioned proximally. However, the proximally positioned Emsold metatarsal dome was found to lead to the best combination plantar pressure redistribution.

\section{Maximum force}

When analysed with the mask that was proximal to the metatarsal heads, where the bulk of the metatarsal dome was positioned, there was a significant effect for maximum force at the forefoot between the 7 conditions 


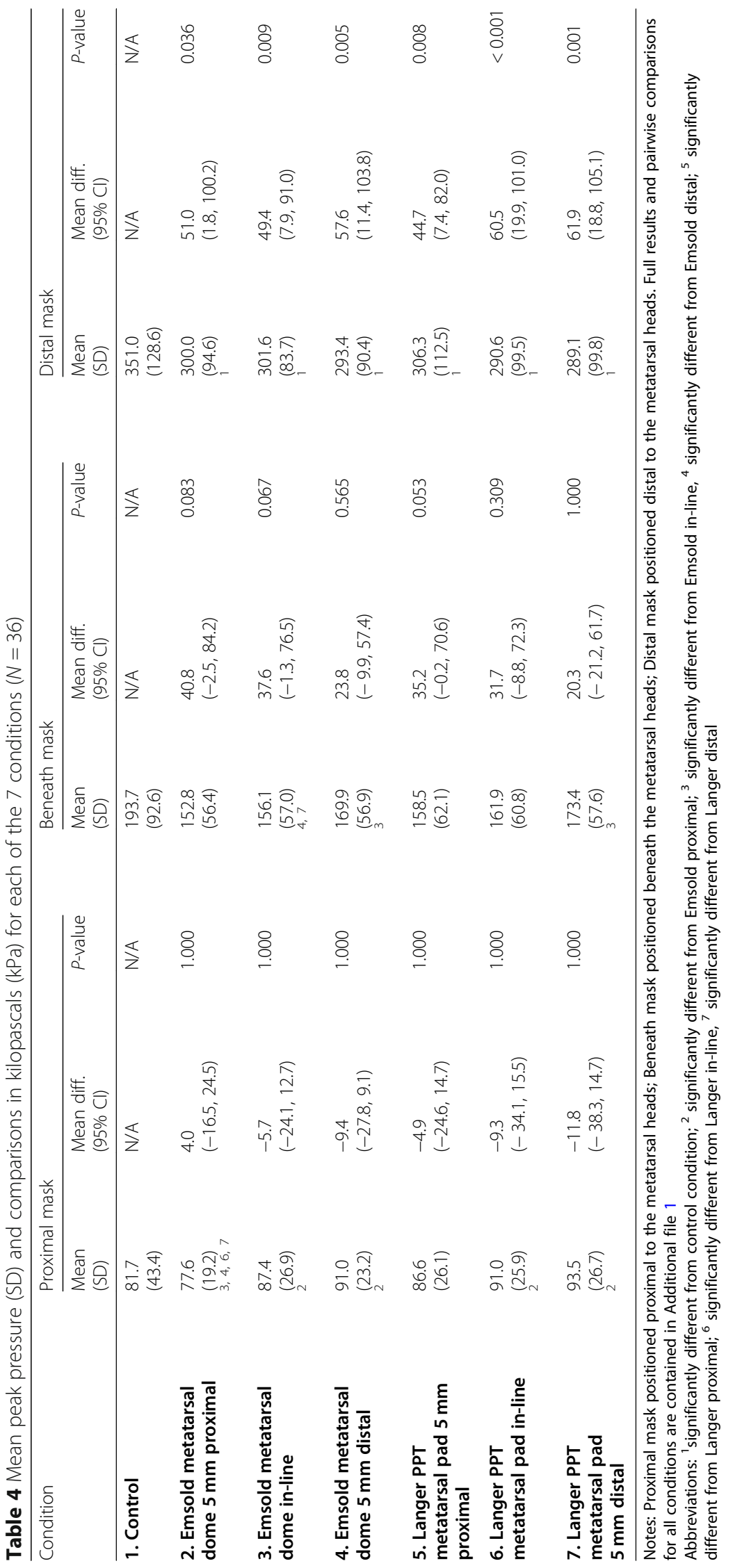



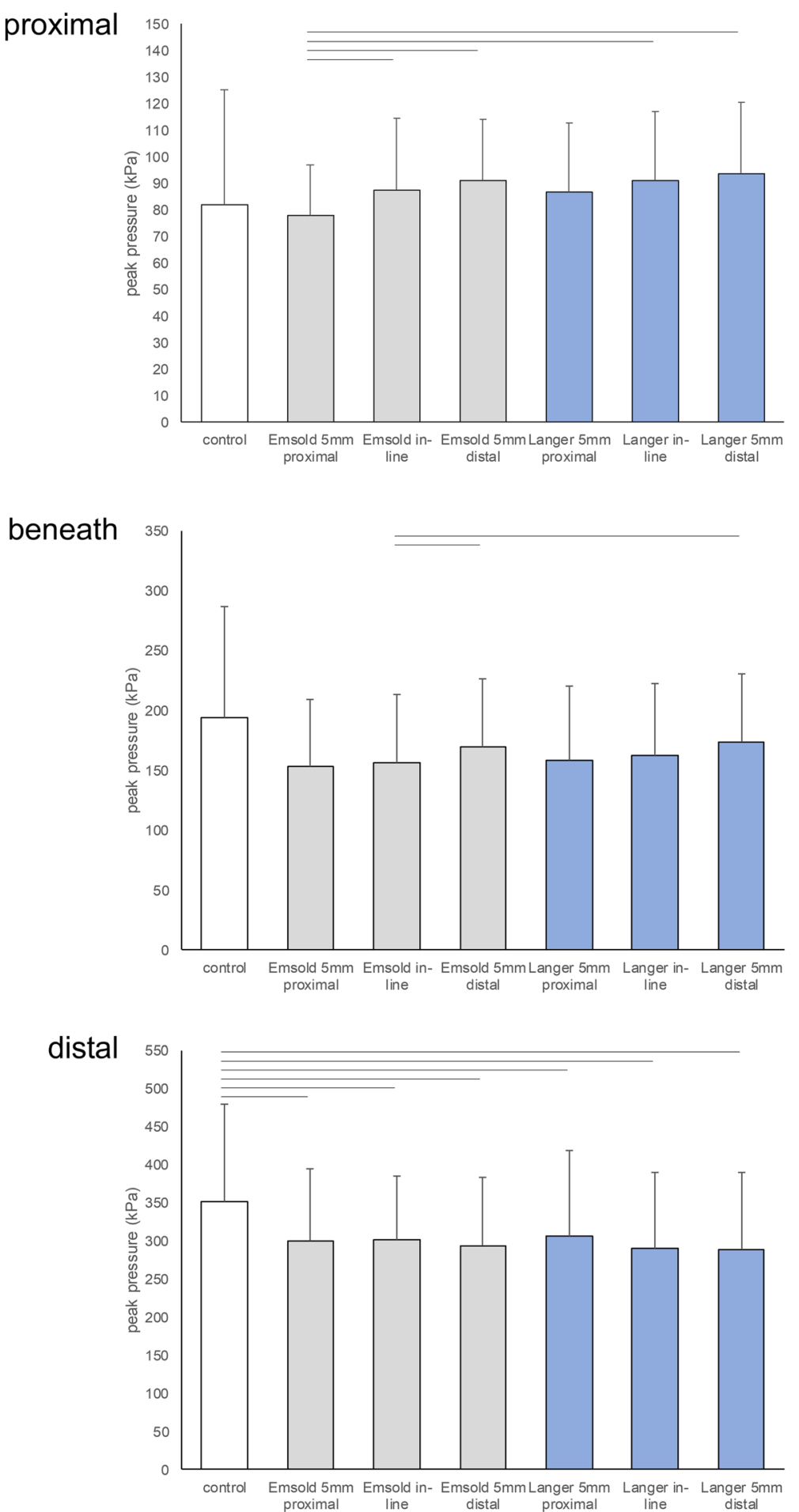

Fig. 3 Graphic presentation of mean peak pressure (SD) in kilopascals ( $\mathrm{kPa}$ ) for each of the 7 conditions for the proximal, beneath and distal masks (bars at the top of each graph represent conditions that were significantly different, $p<0.05$ )

$\left(\mathrm{F}_{2.2,}, 78.4=6.332, p=0.002\right)$. Three of the metatarsal dome conditions significantly increased maximum force when compared to the control condition (Table 5 and
Fig. 4); the in-line positioned Emsold metatarsal dome, the distally positioned Emsold metatarsal dome, and the proximally positioned Langer PPT metatarsal pad. 


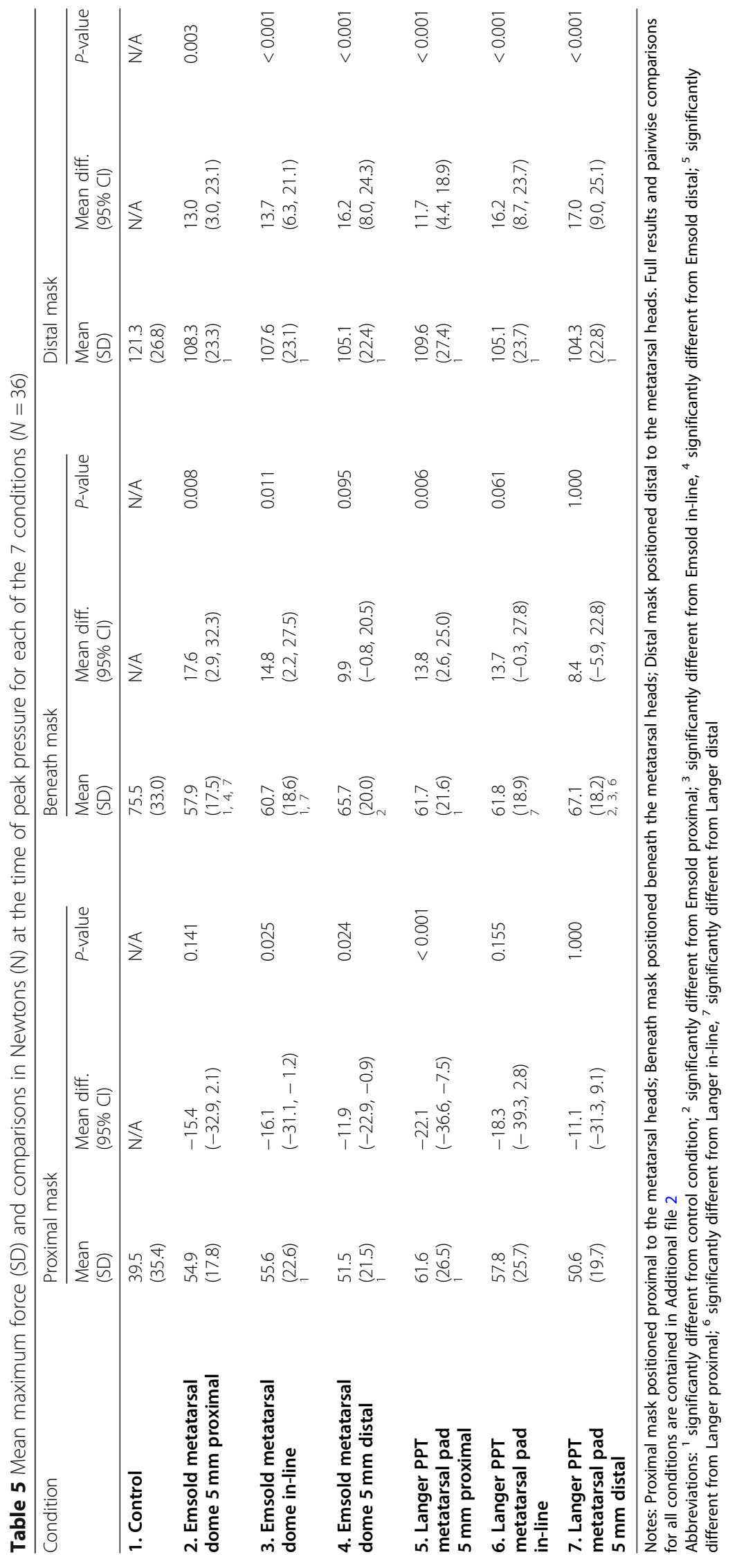



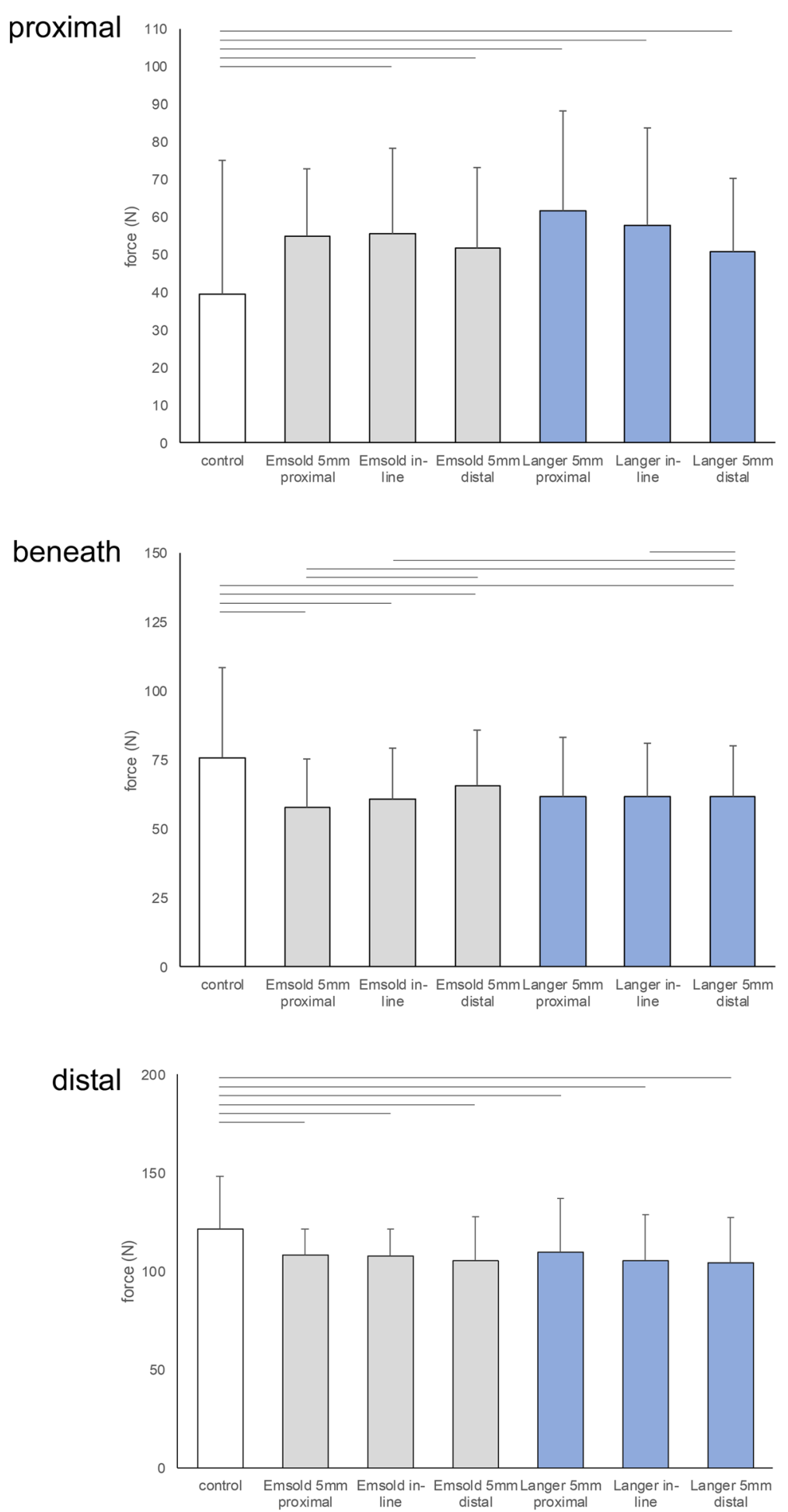

Fig. 4 Graphic presentation of mean force (SD) in Newtons (N) at the time of peak pressure for each of the 7 conditions for the proximal, beneath and distal masks (bars at the top of each graph represent conditions that were significantly different, $p<0.05$ )

There were no significant differences between any of the pad conditions (Additional file 2 presents pairwise comparisons).
When analysed with the mask that was beneath the metatarsal heads mask, there was a significant effect for maximum force at the forefoot between the 7 conditions 
$\left(F_{2.3}, 81.4=9.049, \quad p<0.001\right)$. Three of the metatarsal dome conditions significantly decreased maximum force when compared to the control condition (Table 5 and Fig. 4); the proximally positioned Emsold metatarsal dome, the in-line positioned Emsold metatarsal dome, and the proximally positioned Langer PPT metatarsal pad. There were several significant differences between the metatarsal dome conditions (Additional file 2 presents pairwise comparisons) dependent on the type of metatarsal dome (Emsold or Langer) and the position of the metatarsal dome (proximal, in-line or distal). The proximally positioned Emsold metatarsal dome led to a significantly larger decrease in maximum force when compared to the distally positioned Emsold metatarsal dome and the distally positioned Langer PPT metatarsal pad. In addition, the in-line positioned Emsold metatarsal dome and the in-line positioned Langer PPT led to a significantly larger decrease in maximum force when compared to the distally positioned Langer PPT metatarsal pad.

When analysed with the mask that was distal to the metatarsal heads mask, where the highest forces were recorded, there was a significant effect for maximum force at the forefoot between the 7 conditions $\left(\mathrm{F}_{4.3}, 149.4=\right.$ 13.365, $p<0.001)$. All of the metatarsal dome conditions significantly reduced maximum force when compared to the control condition (Table 5 and Fig. 4), but there were no significant differences between any of the metatarsal dome conditions (Additional file 2 presents pairwise comparisons). This reduction in force was in the order of $12-17 \mathrm{~N}$.

Summarising the maximum force findings, the metatarsal domes significantly reduced maximum force in the mask that was distal to the metatarsal heads, where the force readings were at their highest. For some of the metatarsal dome conditions, this did not occur at the expense of significantly increasing force in the mask that was proximal to the metatarsal heads, where the bulk of the metatarsal dome was positioned; that is, maximum force was not simply moved from one area to another. Overall, the proximally positioned Emsold metatarsal dome was found to lead to the best combination of significantly decreasing maximum force distal to the metatarsal heads, but not adversely increasing maximum force proximally.

\section{Contact area}

When analysed with the mask that was proximal to the metatarsal heads, where the bulk of the metatarsal dome was positioned, there was a significant effect for contact area at the forefoot between the 7 conditions $\left(\mathrm{F}_{3.3}\right.$, $115.5=23.600, p<0.001$ ) (Table 6 and Fig. 5). There were several significant differences between the metatarsal dome conditions (Additional file 3 presents pairwise comparisons) dependent on the type of metatarsal dome (Emsold or Langer) and the position of the metatarsal dome (proximal, in-line or distal). For both the Emsold metatarsal dome and the Langer PPT metatarsal pad, the more proximal the metatarsal dome was positioned, the greater the contact area at the forefoot. The proximally positioned Emsold metatarsal dome led to significantly greater increase in contact area compared to the distally positioned Emsold metatarsal dome and the distally positioned Langer PPT metatarsal pad. The in-line positioned Emsold metatarsal dome led to significantly greater increase in contact area compared to the distally positioned Langer PPT metatarsal pad. The proximally positioned Langer PPT metatarsal pad led to significantly greater increase in contact area compared to the distally positioned Emsold metatarsal dome and the distally positioned Langer PPT metatarsal pad. The in-line positioned Langer PPT metatarsal pad led to significantly greater increase in contact area compared to the distally positioned Langer PPT metatarsal pad. These increases in contact area relative were in the order of 2-4 $\mathrm{cm}^{2}$.

There were no significant effects for contact area at the forefoot between the 7 conditions when analysed with the mask that was beneath to the metatarsal heads $\left(\mathrm{F}_{4.6,159.6}=1.840, p=0.115\right)$ and with the mask that was distal to the metatarsal heads $\left(\mathrm{F}_{6.0},{ }_{210.0}=1.448\right.$, $p=0.198)$. These results are presented in Table 6 and Fig. 5 (Additional file 3 presents pairwise comparisons).

Summarising the contact area findings, the metatarsal domes significantly increased contact area in the mask that was proximal to the metatarsal heads, where the bulk of the metatarsal dome was positioned. The proximally positioned Emsold metatarsal dome and the proximally positioned Langer PPT metatarsal pad led to the largest increases in contact area at the forefoot.

\section{Discussion}

The aim of this study was to evaluate the effect on plantar pressure of metatarsal domes (the Emsold metatarsal dome and the Langer PPT metatarsal pad) in different positions in older people with a history of forefoot pain. To achieve this, and to help explain how the metatarsal domes produce the effects observed, we first developed a new anatomically-based masking protocol [30], which separated the forefoot into three distinct mask regions (proximal to the metatarsal heads, beneath the metatarsal heads, and distal to the metatarsal heads). We chose to develop this method because in a previous study that we conducted [12], which evaluated the effects of different forefoot pads on plantar pressure, we could not accurately determine where in the forefoot peak pressure was reduced, nor could we adequately explain how the metatarsal domes achieved their effect. 


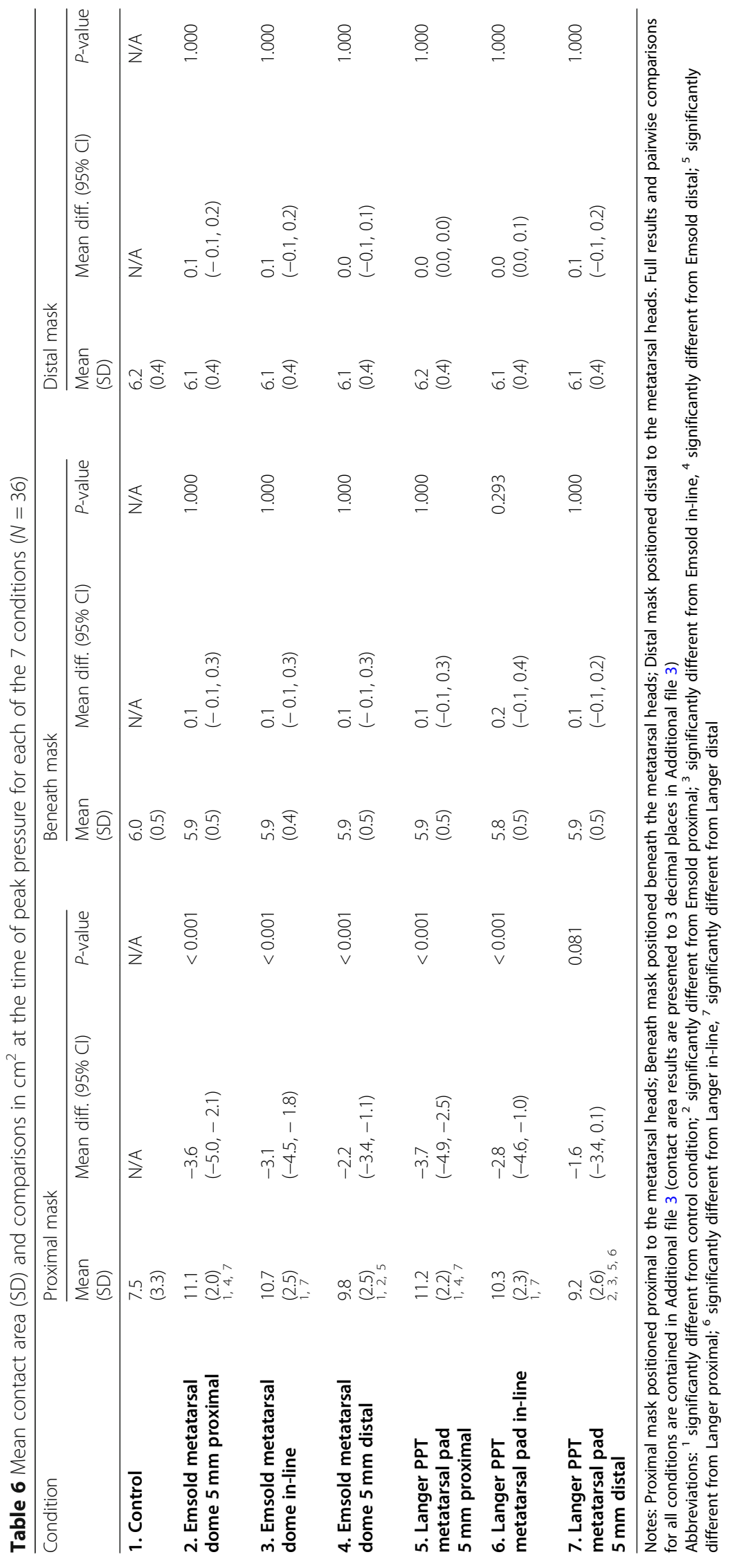



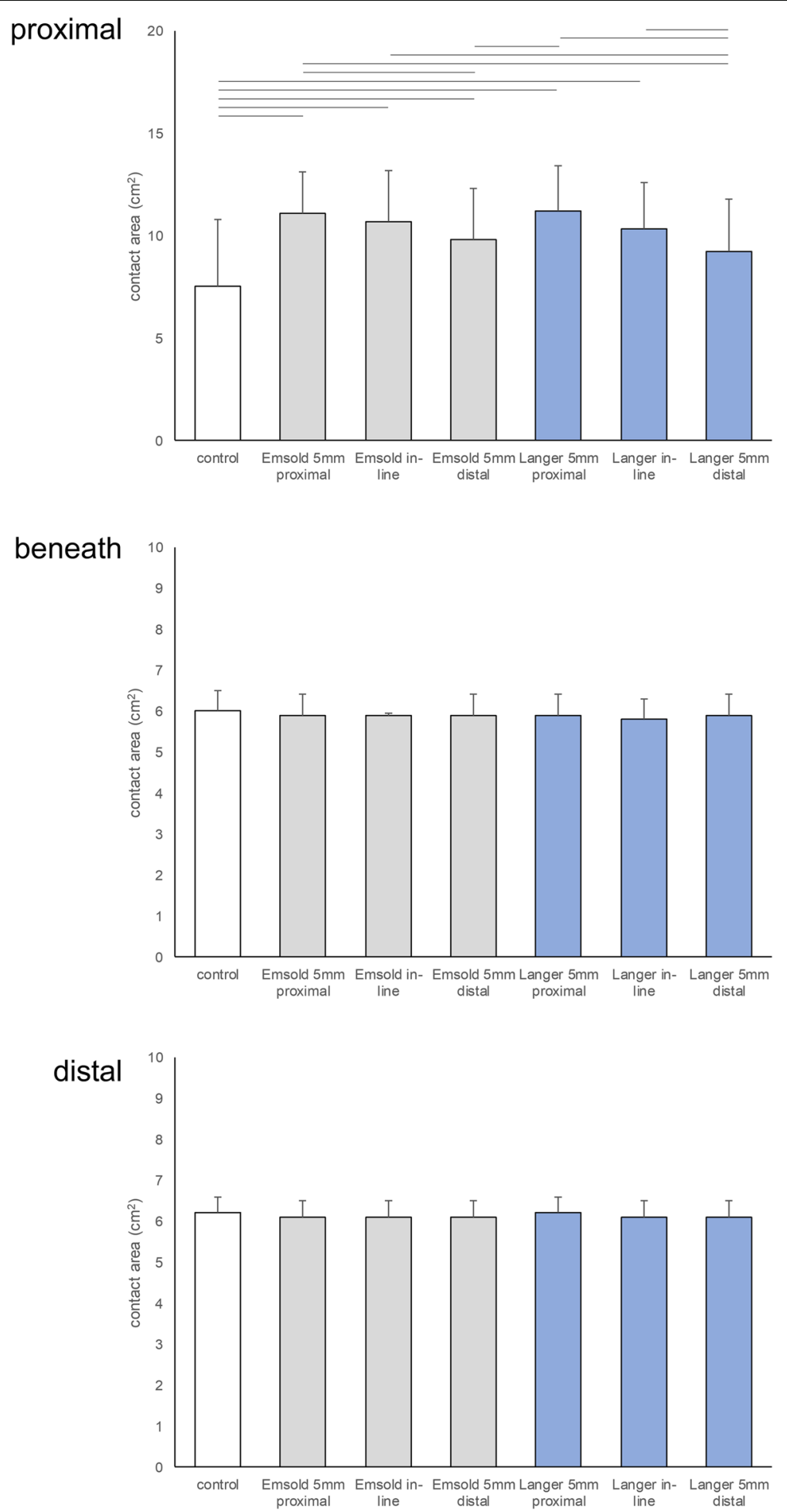

Fig. 5 Graphic presentation of mean contact area (SD) in $\mathrm{cm}^{2}$ at the time of peak pressure for each of the 7 conditions for the proximal, beneath and distal masks (bars at the top of each graph represent conditions that were significantly different, $p<0.05$ )

The results of our current study show that the metatarsal domes did reduce forefoot plantar pressure in comparison to the control (no metatarsal dome) condition, and this was most notable - in the order of $45-60 \mathrm{kPa}$ - under the area of the forefoot where the highest plantar pressures were recorded, which was 
distal to the metatarsal heads. This reduction in plantar pressure should be beneficial where high plantar pressures are associated with forefoot pain. However, this beneficial offloading may be negated if at the same time the metatarsal dome adversely increases plantar pressure more proximally where the bulk of the metatarsal dome is positioned. With this in mind, the best combination of offloading is where a metatarsal dome significantly reduces plantar pressure distally, where the highest plantar pressures are, but does not significantly increase plantar pressures proximally, where the bulk of the metatarsal dome is positioned. Our data demonstrates that two of the metatarsal dome conditions achieved this - the Emsold metatarsal dome positioned proximally and the Langer PPT metatarsal pad positioned proximally. Although, when our data for the proximally positioned metatarsal domes is more closely evaluated, the Emsold metatarsal dome was more effective than the Langer PPT metatarsal pad.

This superior performance of the Emsold metatarsal dome compared to the Langer PPT metatarsal pad can be explained by the hardness of the two metatarsal domes. The Emsold metatarsal dome (Shore A hardness of 11 durometer) is relatively 'softer' than the Langer PPT metatarsal pad (Shore A hardness of 20 durometer). This relative softness leads to a better combination of plantar pressure reduction distally but not causing an adverse increase in plantar pressure proximally. Put simply, the softer Emsold metatarsal dome may subtly mould more to the foot in the region proximal to the metatarsal heads, thus causing no increase in plantar pressure at this site, while still decreasing plantar pressure where it is most needed, which is distal to the metatarsal heads where the highest plantar pressures are found. It is plausible that this characteristic of the Emsold metatarsal dome would be important for comfort and wearability, although further studies are needed to determine this for certain.

Interestingly, while all the metatarsal dome conditions that we tested were found to decrease plantar pressure distally, four significantly increased plantar pressure proximally, which as inferred previously, may lead to comfort issues when using the metatarsal domes in these positions (i.e. potential irritation of the skin adjacent to where the pad is positioned). These conditions included the Emsold metatarsal dome and Langer PPT metatarsal pad when positioned in-line or distal to the metatarsal heads. These findings indicate that metatarsal domes should not be positioned in-line or distal to the metatarsal heads; instead, the best position for them is where their anterior border is proximal to the metatarsal parabola (i.e. the line representing the metatarsal heads). In our study this position was $5 \mathrm{~mm}$ proximal to the metatarsal parabola, so until further studies are done that indicate otherwise, we recommend this position as being the most effective position.

The anatomically-based masking protocol that we used for this study [30] provided data to enhance our understanding of the mechanism of action of the metatarsal dome. To achieve a reduction in plantar pressure, forefoot pads must reduce force and/or increase contact area on the plantar surface of the foot $[20,21]$. We found that the metatarsal domes tested in our current study, particularly when positioned proximal to the metatarsal heads, reduced plantar pressure in the forefoot by both decreasing force and increasing contact area. They achieve this by the shape and location of the pad, which reduces force distal to the second, third and fourth metatarsal heads by redistributing some of that force proximally. The pad achieves this by increasing the area over which the force is distributed. Because pressure is equal to force divided by area, any decrease in force or increase in contact area by the metatarsal dome will lead to a decrease in plantar pressure at the forefoot. While this finding is intuitive, this study is the first to demonstrate this in a systematic way by using the anatomicallybased masking protocol.

The finding that more proximally positioned forefoot pads reduce plantar pressure more than distally positioned forefoot pads contrasts with our earlier study [12]. In that study, we found that a Langer PPT metatarsal pad positioned $5 \mathrm{~mm}$ distal to the metatarsal heads reduced plantar pressure more in the forefoot than a similar pad positioned $10 \mathrm{~mm}$ proximal to the metatarsal heads. This finding was most likely due to the plantar pressure masking technique used in that study, which was a single, relatively large mask that represented the entire forefoot, rather than the three smaller anatomically-based masks that we used in the current study (however, also note that in the current study we positioned this pad $5 \mathrm{~mm}$ proximal to the metatarsal heads, not $10 \mathrm{~mm}$ proximal). The anatomically-based masking protocol, therefore, enables a better explanation, in a dose-response manner, of the changes in plantar pressure (i.e. force and contact area) between various positions of metatarsal domes. Put simply, the simple forefoot mask protocol that we used in our earlier study did not provide us with the ability to distinguish more subtle plantar pressure changes with the forefoot pads compared to the anatomically-based masking protocol that we used in our current study.

Reductions in plantar pressure measured in our study were in the order of $45-60 \mathrm{kPa}$ or approximately 13 to $17 \%$ (distal mask analysis), which is similar to what we found in our earlier study [12]. Similar reductions have also been found in other in-shoe plantar pressure studies of forefoot padding $[10,13,15,22,31-33]$, although some of the pads in these studies are somewhat different 
to the pads we used and were tested on different populations (e.g. participants with diabetes or rheumatoid arthritis). This highlights that our new anatomically-based masking protocol is capable of detecting plantar pressure changes of similar magnitude to other protocols, but it has the added benefit of being able to explain how these plantar pressure changes are produced.

It has been shown that redistributing plantar pressure in the forefoot can lead to a reduction in forefoot pain [4]. However, it is not known whether a difference of approximately 13 to $17 \%$ - the reductions found in this study - is sufficient to reduce pressure-related forefoot pain. One study [11] reported that pain relief occurred when plantar pressure was reduced by approximately $12 \%$, however this study was performed on a younger population (mean age approximately 50 years compared to the mean age of our sample of approximately 75 years). Randomised trials where both plantar pressure and pain are measure simultaneously are needed to ascertain this.

This study has four strengths. Firstly, previous studies have investigated the effects of different positions of metatarsal pads [13-15], however none of these studies have specifically been conducted on older people with forefoot pain. Secondly, this study compared commercially available prefabricated metatarsal domes of different densities (i.e. hardnesses), and as such, our findings reflect clinical practice. However, our study is difficult to compare to others as they used metatarsal domes of different densities to the ones used in our study, and some studies used pads made from other materials such as cork, felt or foam [13-15]. Thirdly, we elected to perform data analysis using a new anatomically-based masking protocol for the forefoot region (rather than mask the entire forefoot), which provided us with the ability to explain more effectively the mechanism of action of the forefoot pads. Further, our new masking technique has an advantage over attempting to mask individual metatarsal heads because of the poor reliability associated of that technique [34] and because older people having a high level of forefoot deformities making determination of the position of individual metatarsal heads difficult $[1,35,36]$. Finally, the methods of our study were pragmatic as the protocol for the placement of forefoot pads was designed to reflect clinical practice.

There are, however, two limitations to this study that should be taken into account. Firstly, this study only evaluated plantar pressure, and although increased plantar pressure has been associated with forefoot pain in older people, randomised trials using patient-reported outcome measures are needed to evaluate whether forefoot pads do indeed reduce forefoot pain in older people, and to determine whether they are comfortable and wearable. Secondly, although the pedar- $\mathrm{X}$ has been shown to be a valid and reliable plantar pressure system it only records forces that are applied perpendicular to the pressure sensors [25-28]. Accordingly, the shear component of forces acting at the interface between the metatarsal pad and the sensor is unable to be determined [37, 38]. Furthermore, the contact surface of the forefoot pads that were used in this study are curvilinear but the sensors are calibrated when placed flat. As a result, it is possible that inherent measurement error occurs, but the magnitude of any such error is currently unknown. Potential accuracy errors have been shown to exist with plantar measuring systems when measuring contact area, otherwise referred to as spatial resolution [39-41]. Despite these limitations, in-shoe pressure measuring systems are considered the best available method for measuring forces acting between in-shoe devices and the foot $[37,38]$ and they have been in common use in the last two decades to evaluate the mechanical effects of in-shoe devices and shoes in older populations $[12,19,29,42]$, as well as in people with degenerative disorders [43, 44], rheumatoid arthritis [23, 31] and diabetic peripheral neuropathy $[45,46]$.

\section{Conclusion}

The results of this study indicate that metatarsal domes reduce plantar pressure in the forefoot in older people with a history of forefoot pain. All metatarsal dome conditions significantly reduced peak pressure in the forefoot, however metatarsal domes that were positioned $5 \mathrm{~mm}$ proximal to the metatarsal heads provided the best balance of reducing plantar pressure distal to the metatarsal heads, where the pressure is at its greatest, but not adversely increasing pressure proximally where the bulk of the pad is positioned. When positioned $5 \mathrm{~mm}$ proximal to the metatarsal heads, the Emsold metatarsal dome was more effective than the Langer PPT metatarsal pad, so we cautiously recommend this forefoot pad for alleviating plantar pressure in older people with a history of forefoot pain.

\section{Supplementary information}

Supplementary information accompanies this paper at https://doi.org/10. 1186/s13047-020-00388-x.

Additional file 1. Pairwise comparisons for mean peak pressure $(\mathrm{kPa})$. Additional file 2. Pairwise comparisons for mean force $(\mathrm{N})$ at time of peak pressure.

Additional file 3. Pairwise comparisons for contact area $\left(\mathrm{cm}^{2}\right)$ at time of peak pressure.

\section{Acknowledgements}

Miss Xiling Jerilyn Tan for assistance with data collection. We also gratefully acknowledge Briggate Medical Company, Braeside, Victoria, Australia for supplying the metatarsal pads used in this study for no cost.

Authors' contributions

$\mathrm{KBL}$ led the inception and design of the study, assisted with recruitment, conducted the statistical analysis, and drafted the manuscript. CAA participated in the inception and design of the study, undertook participant 
recruitment and collected data, contributed to statistical analysis, and assisted drafting the manuscript. DRB participated in the inception and design of the study, provided technical support for and calibrated the pedar ${ }^{\oplus} \mathrm{X}$ in-shoe plantar pressure system, and assisted drafting the manuscript. HBM participated in the inception and design of the study, assisted with recruitment, and assisted drafting the manuscript, including production of the graphs and figures. SF developed the anatomically-based masking protocol used to analyse the data, conducted the statistical analysis, and assisted drafting the manuscript. All authors read and approved the final manuscript.

\section{Funding}

There is no funding to declare regarding this study.

\section{Availability of data and materials}

The datasets used and/or analysed during the current study are available from the corresponding author upon reasonable request.

\section{Ethics approval and consent to participate}

Ethics approval was obtained from the La Trobe University Faculty Human Ethics Committee - application FHEC12/207. All participants signed written informed consent prior to recruitment into the study.

\section{Consent for publication}

All participants (via the informed consent process) provided consent to publish data collected in this study providing they could not be identified.

\section{Competing interests}

DRB is currently in the position of Associate Editor of the Journal of Foot and Ankle Research. It is journal policy that editors are removed from the peer review and editorial decision-making processes for papers they have coauthored. KBL, DRB, HBM and SF also developed the new anatomically-based masking protocol that was used to analyse the data in this study.

\section{Author details}

${ }^{1}$ Discipline of Podiatry, School of Allied Health, Human Services and Sport, La Trobe University, Melbourne, Victoria 3086, Australia. ${ }^{2}$ La Trobe Sport and Exercise Medicine Research Centre, La Trobe University, Melbourne, Victoria 3086, Australia. ${ }^{3}$ Musculoskeletal Research Center, School of Rehabilitation Sciences, Isfahan University of Medical Sciences, Isfahan, Iran. ${ }^{4}$ Centre for Health Sciences Research, University of Salford, Salford M5 4WT, England.

\section{Received: 13 February 2020 Accepted: 24 April 2020}

Published online: 06 May 2020

\section{References}

1. Dunn JE, Link CL, Felson DT, Crincoli MG, Keysor JJ, McKinlay JB. Prevalence of foot and ankle conditions in a multiethnic community sample of older adults. Am J Epidemiol. 2004;159:491-8.

2. Mølgaard C, Lundbye-Christensen S, Simonsen O. High prevalence of foot problems in the Danish population: a survey of causes and associations. Foot (Edinb). 2010;20:7-11.

3. Thomas MJ, Roddy E, Zhang W, Menz HB, Hannan MT, Peat GM. The population prevalence of foot and ankle pain in middle and old age: a systematic review. Pain. 2011;152:2870-80.

4. Mickle KJ, Munro BJ, Lord SR, Menz HB, Steele JR. Foot pain, plantar pressures, and falls in older people: a prospective study. J Am Geriatr Soc. 2010;58:1936-40.

5. Mickle KJ, Munro BJ, Lord SR, Menz HB, Steele JR. Gait, balance and plantar pressures in older people with toe deformities. Gait Posture. 2011;34:347-51.

6. Hill CL, Gill TK, Menz HB, Taylor AW. Prevalence and correlates of foot pain in a population-based study: the north West Adelaide health study. J Foot Ankle Res. 2008;1:2.

7. Menz HB. Foot problems in older people: assessment and management. Edinburgh: Churchill Livingstone; 2008.

8. Bardelli M, Turelli L, Scoccianti G. Definition and classification of metatarsalgia. Foot Ankle Surg. 2003;9:79-85.

9. Menz HB, Fotoohabadi MR, Munteanu SE, Zammit GV, Gilheany MF. Plantar pressures and relative lesser metatarsal lengths in older people with and without forefoot pain. J Orthop Res. 2013;31:427-33.
10. Jackson L, Binning J, Potter J. Plantar pressures in rheumatoid arthritis using prefabricated metatarsal padding. J Am Podiatr Med Assoc. 2004;94:239-45.

11. Kang J-H, Chen M-D, Chen S-C, Hsi W-L. Correlations between subjective treatment responses and plantar pressure parameters of metatarsal pad treatment in metatarsalgia patients: a prospective study. BMC Musculoskelet Disord. 2006;7:95.

12. Lee $\mathrm{P}$, Landorf $\mathrm{K}$, Bonanno $\mathrm{D}$, Menz $\mathrm{H}$. Comparison of the pressure-relieving properties of various types of forefoot pads in older people with forefoot pain. J Foot Ankle Res. 2014;7:18

13. Hayda R, Tremaine MD, Tremaine K, Banco S, Teed K. Effect of metatarsal pads and their positioning: a quantitative assessment. Foot Ankle Int. 1994; 15:561-6.

14. Hsi WL, Kang JH, Lee XX. Optimum position of metatarsal pad in metatarsalgia for pressure relief. Am J Phys Med Rehabil. 2005;84:514-20.

15. Hastings MK, Mueller MJ, Pilgram TK, Lott DJ, Commean PK, Johnson JE. Effect of metatarsal pad placement on plantar pressure in people with diabetes mellitus and peripheral neuropathy. Foot Ankle Int. 2007;28:84-8.

16. Spink M, Menz H, Lord S. Efficacy of a multifaceted podiatry intervention to improve balance and prevent falls in older people: study protocol for a randomised trial. BMC Geriatr. 2008;8:30.

17. Spink MJ, Menz HB, Fotoohabadi MR, Wee E, Landorf KB, Hill KD, Lord SR. Effectiveness of a multifaceted podiatry intervention to prevent falls in community dwelling older people with disabling foot pain: randomised controlled trial. BMJ. 2011;342:d3411.

18. Friedman LM, Furberg CD. DeMets DL. Fundamentals of clinical trials. New York: Springer; 2010.

19. Bonanno DR, Landorf KB, Menz HB. Pressure-relieving properties of various shoe inserts in older people with plantar heel pain. Gait Posture. 2011;33: 385-9.

20. Redmond A, Lumb P, Landorf K. Effect of cast and noncast foot orthoses on plantar pressure and force during normal gait. J Am Podiatr Med Assoc. 2000;90:441-9.

21. Burns J, Crosbie J, Ouvrier R, Hunt A. Effective orthotic therapy for the painful cavus foot: a randomized controlled trial. J Am Podiatr Med Assoc. 2006;96:205-11.

22. Guldemond NA, Leffers $P$, Schaper NC, Sanders AP, Nieman F, Willems $P$, Walenkamp GHIM. The effects of insole configurations on forefoot plantar pressure and walking convenience in diabetic patients with neuropathic feet. Clin Biomech (Bristol, Avon). 2007;22:81-7.

23. Hennessy K, Burns J, Penkala S. Reducing plantar pressure in rheumatoid arthritis: A comparison of running versus off-the-shelf orthopaedic footwear. Clin Biomech (Bristol, Avon). 2007;22:917-23.

24. Redmond A, Landorf K, Keenan A-M. Contoured, prefabricated foot orthoses demonstrate comparable mechanical properties to contoured, customised foot orthoses: a plantar pressure study. J Foot Ankle Res. 2009;2:20.

25. Hsiao H, Guan J, Weatherly M. Accuracy and precision of two in-shoe pressure measurement systems. Ergonomics. 2002;45:537-55.

26. Murphy DF, Beynnon BD, Michelson JD, Vacek PM. Efficacy of plantar loading parameters during gait in terms of reliability, variability, effect of gender and relationship between contact area and plantar pressure. Foot Ankle Int. 2005;26:171-9.

27. Putti AB, Arnold GP, Cochrane L, Abboud RJ. The Pedar ${ }^{\oplus}$ in-shoe system: repeatability and normal pressure values. Gait Posture. 2007;25:401-5.

28. Ramanathan AK, Kiran P, Arnold GP, Wang W, Abboud RJ. Repeatability of the Pedar- $X^{\circledR}$ in-shoe pressure measuring system. Foot Ankle Surg. 2010;16:70-3.

29. Cronkwright DG, Spink MJ, Landorf KB, Menz HB. Evaluation of the pressureredistributing properties of prefabricated foot orthoses in older people after at least 12 months of wear. Gait Posture. 2011;34:553-7.

30. Forghany S, Bonanno DR, Menz HB, Landorf KB. An anatomically-based masking protocol for the assessment of in-shoe plantar pressure measurement of the forefoot. J Foot Ankle Res. 2018;11:31.

31. Hodge MC, Bach TM, Carter GM. Novel Award First Prize Paper. Orthotic management of plantar pressure and pain in rheumatoid arthritis. Clin Biomech (Bristol, Avon). 1999;14:567-75.

32. Koenraadt KLM, Stolwijk NM, van den Wildenberg D, Duysens J, Keijsers NLW. Effect of a metatarsal pad on the forefoot during gait. J Am Podiatr Med Assoc. 2012;102:18-24.

33. Mueller MJ, Lott DJ, Hastings MK, Commean PK, Smith KEM, Pilgram TK. Efficacy and mechanism of orthotic devices to unload metatarsal heads in people with diabetes and a history of plantar ulcers. Phys Ther. 2006;86:833-42. 
34. Deschamps K, Birch I, Mc Innes J, Desloovere K, Matricali GA. Inter- and intra-observer reliability of masking in plantar pressure measurement analysis. Gait Posture. 2009;30:379-82.

35. Menz HB, Lord SR. Foot pain impairs balance and functional ability in community-dwelling older people. J Am Podiatr Med Assoc. 2001;91:222-9.

36. Garrow AP, Silman AJ, Macfarlane GJ. The Cheshire foot pain and disability survey: a population survey assessing prevalence and associations. Pain. 2004;110:378-84.

37. Orlin MN, McPoil TG. Plantar pressure assessment. Phys Ther. 2000;80:399-409.

38. Spooner SK, Smith DK, Kirby KA. In-shoe pressure measurement and foot orthosis research: a giant leap forward or a step too far? J Am Podiatr Med Assoc. 2010;100:518-29.

39. Urry S. Plantar pressure-measurement sensors. Meas Sci Technol. 1999;10:R16-32.

40. Urry SR, Wearing SC. The accuracy of footprint contact area measurements: relevance to the design and performance of pressure platforms. Foot (Edinb). 2001;11:151-7.

41. Urry SR, Wearing SC. Arch indexes from ink footprints and pressure platforms are different. Foot (Edinb). 2005;15:68-73.

42. Lane TJ, Landorf KB, Bonanno DR, Raspovic A, Menz HB. Effects of shoe sole hardness on plantar pressure and comfort in older people with forefoot pain. Gait Posture. 2014;39:247-51.

43. Rao S, Baumhauer JF, Becica L, Nawoczenski DA. Shoe inserts alter plantar loading and function in patients with midfoot arthritis. J Orthop Sports Phys Ther. 2009;39:522-31.

44. Jannink M, van Dijk H, ljzerman M, Groothuis-Oudshoorn K, Groothoff J, Lankhurst G. Effectiveness of custom-made orthopaedic shoes in the reduction of foot pain and pressure in patients with degenerative disorders of the foot. Foot Ankle Int. 2006;27:974-9.

45. Bus SA, Ulbrecht JS, Cavanagh PR. Pressure relief and load redistribution by custom-made insoles in diabetic patients with neuropathy and foot deformity. Clin Biomech (Bristol, Avon). 2004;19:629-38

46. Raspovic A, Waller K, Wong WM. The effectiveness of felt padding for offloading diabetes-related foot ulcers, at baseline and after one week of wear. Diabetes Res Clin Pract. 2016;121:166-72.

\section{Publisher's Note}

Springer Nature remains neutral with regard to jurisdictional claims in published maps and institutional affiliations.

Ready to submit your research? Choose BMC and benefit from:

- fast, convenient online submission

- thorough peer review by experienced researchers in your field

- rapid publication on acceptance

- support for research data, including large and complex data types

- gold Open Access which fosters wider collaboration and increased citations

- maximum visibility for your research: over $100 \mathrm{M}$ website views per year

At $\mathrm{BMC}$, research is always in progress.

Learn more biomedcentral.com/submissions 